\title{
Diabetic cardiac autonomic neuropathy and arterial stiffness-which comes first?
}

\author{
Serhiyenko VA*, Serhiyenko LM and Serhiyenko AA \\ Department of Endocrinology, Danylo Halytsky Medical University, Lviv, 79010, Ukraine
}

\section{Introduction}

Autonomic dysfunction is a risk factor for cardiovascular diseases (CVDs), however, the exact mechanism linking autonomic dysfunction to CVDs is not known [1]. Cardiac autonomic dysfunction is a wellestablished risk factor for cardiovascular morbidity and all-cause and cardiovascular mortality. Decreased heart rate variability (HRV) and low baroreflex sensitivity (BRS) are considered to be early markers of cardiac autonomic dysfunction [2-5]. Cardiovascular autonomic neuropathy $(\mathrm{CAN})$ is a major risk factor for cardiovascular injury resulting in heart attack, congestive heart failure (HF), stroke as well as sudden arrhythmic death. The latter is associated with myocardial structural remodelling that follows hypertension (HT), such as hypertrophy and fibrosis. This remodelling accompanies changes in expression, distribution, and function of cell membrane ion channels, intercellular gap junction connexin- 43 channels, $\mathrm{Ca} 2+-$-cycling proteins, and extracellular matrix composition $[4,5]$.

Recently, the possibility of using a new CVDs risk factor, which could be more promising in terms of prognosis than metabolic syndrome (MetS), namely, "syndrome of vascular aging", is actively discussed. The processes of increasing the rigidity of the arteries due to the decrease in the content of elastin and the increase in the concentration of collagen in the walls of the vessels. as well as qualitative changes in the arterial wall due to the violation of endothelial-mediated vasodilation, are known in physiological aging, but a number of factors are able to accelerate this process [6-9].

Since aortic stiffness determines its damping function and pulse wave velocity (PWV), and also affects the level of aortic pulse pressure (PPao) and aortic augmentation index (IAxao), these indices may be recommended for its evaluation. Central systolic blood pressure (SBPao) more reflects the blood flow in the coronary and cerebral vessels compared with central diastolic BP (SBPbr) and is a more significant predictor of CVDs. Therefore, measurements of PPao and IAxao may be more important for the characterization of the state of the cardiovascular system than just the evaluation of the rigidity of the vessel wall in terms of PWV [7-9].

Several epidemiological studies reported that increased arterial stiffness (AS) predicts mortality and morbidity, independently of other cardiovascular (CV) risk factors. AS measured through carotid-femoral PWV, a gold standard method has been associated with subclinical CVDs. Furthermore, clinical studies have shown that the AS increases with aging or various pathological processes associated with HT, MetS, chronic renal disease, and diabetes mellitus (DM) [10]. Diabetes may enhance AS through pathological changes in the vascular bed, such as reduced nitric oxide (NO) bioavailability, increased oxidative stress
(OS), chronic low-grade inflammation, increased sympathetic tone and changes in type or structure of elastin and/or collagen in the arterial wall [10-12].

Many of the pathophysiological mechanisms responsible for vascular dysfunction in DM are determined by hyperglycemia, which is associated with the activation of pro-inflammatory transcription factors and increased OS, leading to vasculopathy. Increased advanced glycation end-product (AGEs) levels may alter the important matrix of molecules in the vessel wall. In addition, some studies show endothelial and vascular smooth muscle cell (VSMCs) dysfunction in diabetic individuals compared to controls indicating that T2DM may both reduce the bioavailability of endothelial $\mathrm{NO}$ and attenuate sensitivity of the VSMCs to NO. All these pathways appear to be involved in mediating the hyperglycemia-associated AS. Patients without DM but with primary autonomic failure have stiffer aortas when compared with healthy age- and sex-matched control individuals. These findings imply that there is a pathophysiological link between cardiac autonomic dysfunction and AS and that the preservation of the elastic properties of the arteries strongly depends on the integrity of the autonomic nervous system (ANS) [10,13-14].

The pathophysiological link between aortic stiffness and autonomic dysfunction and whether impaired cardiac autonomic function induces AS or whether increased AS leads to the impairment of the autonomic function remains obscure. Both AS and cardiac autonomic dysfunction share common pathogenetic pathways including chronic hyperglycemia and hyperinsulinemia (HI), formation of AGEs and protein kinase $\mathrm{C}$ (PKC) activation, low grade-inflammation and endothelial dysfunction (ED) [14].

\section{Hyperglycemia}

The hyperglycemia in diabetes leads to vascular and neuronal damages by causing nonenzymatic glycosylation, increased OS and activation of polyol and protein kinase $\mathrm{C}$ pathways. The mechanisms triggered by hyperglycemia result in ED and AS, which are responsible for the microvascular and macrovascular complications of diabetes [15].

${ }^{*}$ Correspondence to: Victoria Serhiyenko, $\mathrm{MD}, \mathrm{PhD}$, Department of Endocrinology, Danylo Halytsky Medical University, Lviv, 79010, Ukraine, Tel: +380-322-769496, Fax: +380-322-769496, E-mail: serhiyenkoa@gmail.com; serhieynko@inbox.ru

Key words: diabetes mellitus, cardiac autonomic neuropathy, hyperglycemia, insulin resistance, inflammation, endothelial dysfunction, heart rate variability, arterial stiffness

Received: July 30, 2018; Accepted: August 06, 2018; Published: August 08, 2018 
Hyperglycemia has been shown to trigger ED through decrease in NO synthesis, increase in free radicals levels, and deterioration of antioxidant defence mechanisms. Chronic glycemic exposure also induces VSMCs proliferation and chronic inflammation, increases generation of AGEs and enhances collagen cross-linking within the arterial wall, up-regulates matrix metalloproteinase- 2 and -9 expression (enzymes degrading elastin), augments the generation of angiotensin-2 in vascular tissue and increases endothelial permeability [16]. ED is considered a key event in the initiation of the atherosclerotic process, yet it also leads to "functional" stiffening of arteries, as a continuous NO release by endothelium contributes to the functional regulation of arterial elasticity, aimed to adapt peripheral conduit artery mechanics to changes in blood flow [17].

\section{Insulin resistance}

Several prospective studies have demonstrated that insulin resistance (IR), as assessed by various techniques, is related to CVDs in both non-diabetic and diabetic subjects, independently of established risk factors. The risk of CVDs events increased across quintiles of the homeostasis model assessment of insulin resistance (HOMA-IR); the association between HOMA-IR index and CVDs was demonstrated. IR has been shown to be associated with decreased synthesis/release of NO and enhanced generation of reactive oxygen species, as well as with an excessive free fatty acids (FFAs) release from adipose tissue. Increased circulating levels of FFAs may impair endothelial function and induce a low-grade inflammation (through activation of nuclear factor kappa-light-chain-enhancer of activated B cells (NF- $\kappa \mathrm{B})[17,18]$. Hyperinsulinemia augments hepatic very-low-density lipoproteins synthesis, increases cholesterol transport/synthesis in cultured arterial SMCs, stimulates the proliferation of arterial SMCs, augments collagen synthesis and turns on multiple genes involved in inflammation [17]. In subjects with type 2 (T2) DM (T2DM), IR and the use of alcohol were associated with increased AS which supports the hypothesis that increased AS can act as a mediating factor in the association between T2DM and increased risk of atherothrombotic disease [19].

It has been shown that fasting insulin levels were associated to mean carotid intima-media thickness (CIMT); insulin sensitivity was negatively associated with CIMT, and this effect was partly explained by traditional $\mathrm{CV}$ risk factors, glucose tolerance and adiposity; the association between HOMA-IR index and CIMT in non-diabetic subjects was fully explained by established CV risk factors, above all by HT; the relationship between HOMA-IR index and carotid atherosclerosis was mostly dependent on the clustered expression of the components of the MetS; the association between lower insulin sensitivity was mediated by circulating FFAs and adipocytokines. These results imply that IR per se has no strong influence on carotid atherosclerosis, and that its effect on carotid wall is mediated by other metabolic, cellular and hemodynamic abnormalities related to the IR syndrome and DM, like dyslipidemia (DLP), FFAs adipocytokines, chronic inflammation and HT [17].

Moreover, a novel relationship between AS, HI and CAN in a T2DM population has been shown in a study which signifies their pathogenic roles in the development of CVDs in diabetic patients [7].

\section{Inflammation}

The mechanisms linking the autonomic imbalance to atherosclerosis are still elusive. Recent studies suggest an association between autonomic function and inflammation in patients with CVDs, showing an inverse relationship between autonomic activity, measured by HRV, and plasma levels of inflammatory markers [1].
Inflammation is a key mediator in the pathophysiology of atherosclerosis involving both the innate and the adaptive immune system. Diabetes mellitus course represents a spectrum, so drawing clear lines between these stages may be inaccurate, and probably the endothelial inflammation and microcirculatory dysfunction starts with the first rises in blood glucose levels $[1,20,21]$. Tumor necrosis factor-alpha (TNF-alpha), interleukin (IL) (IL-1), and some bacterial components like lipopolysaccharides trigger the transcription of a molecule of the selectin family (CD62E) in an NF-kB-dependent signal cascade. CD62E is associated with endothelium of the blood vessel in different different inflammatory situations. Previously published data have demonstrated that E-selectin (CD62) is a proatherogenic and proinflammatory cytokine associated with IR, obesity, and CVDs [22]. It has been suggested that circulating CD62 concentration may be a biomarker for indicating the subsequent development of metabolic diseases and in particular CVDs from a healthy state [23]. Prediabetes patients have higher E-selectin levels as their serum glucose rise, and it looks as they get highest with the DM diagnosis. This is compatible with a hypothesis of rising endothelial inflammation with progression to DM [21].

Previous studies suggest a relationship between autonomic dysfunction and inflammation, as well as between inflammation and atherosclerosis. These findings made us hypothesize that inflammation mediates the atherogenic effects of autonomic dysfunction. Few studies have investigated the whole pathway (autonomic functioninflammation-atherosclerosis) in the same population or carotid atherosclerosis as the primary end-point. Intima media thickness (IMT), a marker of preclinical atherosclerosis, was recently associated with increased C-reactive protein (CRP) and reduced HRV in patients suffering from depression [1]. Ulleryd MA et al. (2017) hypothesize that inflammation can be a mediator in the link between autonomic dysfunction and atherosclerosis, leading to CVDs like stroke and myocardial infarction; reduced autonomic function will increase low-grade inflammation, subsequently worsening the progression of atherosclerosis [1].

Increased CIMT reflects very early atherosclerotic changes, whereas plaque presence indicates a more advanced atherosclerotic process. T2DM patients have higher CIMT (on average by $130 \mu \mathrm{m}$ ), higher prevalence of carotid plaques and higher plaque volume as compared to controls, and CIMT and carotid plaque prevalence have been shown to be associated with fasting plasma glucose levels, glucose fluctuation or glycated $\mathrm{HbAlc}(\mathrm{HbAlc})$, both in non-diabetic and in diabetic populations [17].

\section{Endothelial dysfunction}

$\mathrm{ED}$ and atherosclerosis play an important role in the pathogenesis of end-organ damage. The endothelial layer has many functions, such as homeostasis, vascular permeability, vasoregulation, angiogenesis and inflammatory response. It is widely accepted that ED is the atherosclerosis precursor [24]. DM candidates have an increasing $\mathrm{ED}$ and inflammation with rising blood glucose levels, through the diagnosis of T2DM. ED is a mostly recognized part of macrovascular and microvascular disease complications in diabetes. ED may also precede diabetes onset and may promote IR and glucose dysregulation leading to DM, but there is still controversy about the exact role of ED in patients with prediabetes and DM [21].

In diabetic patients, impaired endothelial functions and increased arterial wall thickness potentially lead to diabetic micro- and macrovascular complications. DM leads to AS by causing functional 
and structural changes in large vessels. Therefore, it is crucial to maintain ED and arterial elasticity by means of intensive diabetes treatment and improved glycemic control. Additionally, management of ED and AS with certain experimental drugs and/or some medication used in clinical practice is promising regarding prevention of diabetic complications. Furthermore, management of ED and AS is likely to decrease the incidence of cardiovascular disease, which is an important cause of mortality in DM [15].

\section{Heart rate variability}

In T2DMs cardiac autonomic dysfunction, expressed as reduced vagal activity, leads to heart rate (HR) acceleration and thus to diastole shortening, but it seems that cardiac autonomic dysfunction may shorten diastole duration (DD) per se, independently of the effect on HR. Since DD influences strongly subendocardial myocardial viability (SVI), cardiac autonomic dysfunction plays a primary role in addition to AS in the impairment of SVI and may thus worsen cardiovascular prognosis [25].

Chorepsima S et al. (2017) showed that beyond BP, impaired cardiac autonomic function assessed by determination of HRV was a significant determinant of abnormal PWV in patients with T2DM. Furthermore, lower values of the frequency-dependent domains of the HRV were independently associated with higher odds of abnormal PWV [14]. It has been shown that low HRV is associated with increased mortality in patients with ischemic heart disease or DM. HRV is also a sensitive indicator of BRS control, specifically the vagal control. Therefore, AS may affect BRS and thereby, HRV. Increased AS evaluated by PWV and/or AISA has been associated with the presence of coronary atherosclerosis and worse cardiovascular prognosis both in general population and specific disease groups, including DM. Decreased HRV in the uncomplicated diabetes patients highlights the obscure process of CAN in diabetic patients that begins even before clinical atherosclerotic CVDs becomes apparent [7]. It has also been shown that surrogate atherosclerosis markers were associated with lower HRV, and increased CIMT in T2DM participants was significantly associated with decreased HRV, independent from conventional CV risk factors. Therefore, the presence of CAN should be considered much earlier in the course of DM, rather than after the development of clinical CVDs [7].

\section{Arterial stiffness}

Increased AS is a common indicator for the atherosclerotic involvement of the vascular system and is known to occur as a result of atherosclerotic risk factors, such as DM, smoking, HT, DLP, and aging. Increased AS is also associated with coronary artery disease (CAD), cerebrovascular disease and peripheral arterial disease [21,24]. These data provide an evidence that diabetes candidates have an increasing $\mathrm{ED}$ and inflammation with rising blood glucose levels, through the diagnosis of T2DM. ED is a mostly recognized part of macrovascular and microvascular disease complications in DM. ED may also precede $\mathrm{DM}$ onset and may promote IR and glucose dysregulation leading to $\mathrm{DM}$, but there is still controversy about the exact role of ED in patients with prediabetes and DM [21]. PWV is the gold-standard method for the assessment of AS and is an independent predictor of CV morbidity and mortality [26]. A number of studies have shown that AS is increased in people with DM and that PWV independently predicts mortality in this group of patients [14]. Reported that, mean IAxao was similar among T2DM patients and non-diabetes controls; however, IAxao was related to diabetes status after multiple adjustments of several risk factors in the regression model. Other studies have reported that IAxao, which was similar between DM and non-diabetes subjects, increased in diabetes patients after adjustment of HR [27]. Yeboah K et al (2016) hypothesize that, compared to age- and gender-match controls, nonhypertensive, nonobese T2DM patients would have higher levels of AS [27]. Blood pressure and impaired cardiac autonomic function are the main determinants of abnormal PWV in people with T2DM, while the association between impaired cardiac autonomic function and AS is not mediated by low BRS or increased HR. Chorepsima S et al (2017) shown that CAN activity influences AS and it should be monitored and reported in studies examining factors affecting PWV [14]. DM was associated with a lower peripheral AS, opposite from the associations seen for carotid-femoral (cfPWV) and brachial-ankle PWV (baPWV) [26].

Despite such stability, in the sixth ten human life, there is a gradual fragmentation of elastin fibers and plates, which is accompanied by changes in the extracellular matrix in the form of overproduction of collagen and deposition of $\mathrm{Ca} 2+$. This diffuse degenerative process leads to increased stiffness of the vessels of the elastic type and the growth of PP [28]. The relationship between the effects of age and hypertonic changes on the vascular wall is extremely complicated. This is due to the fact that a number of pathophysiological changes are unidirectional (increase in the content of collagen), while others have multidirectional effects, which complicates the interpretation of the results of the examination of patients. In particular, vascular ductility may increase with HT and decreases with age. In addition to HT, many traditional risk factors may contribute to the increased rigidity of the vessels, including DLP, smoking, hyperglycemia, etc [28]. Loehr LR et al. (2016) hypothesize that the arterial wall tissue remodeling that occurs with DM may differ pathophysiologically in muscular versus elastic large arteries and that loss of elasticity and recoil does not necessarily translate into stiffening of the muscular arteries [26]. Tsuchikura $S$ et al. (2010) found opposing directions of effect for the association of peripheral and central stiffness with CAD [29] including in a subset of participants with T2DM. Similarly, a recent publication from the ARICstudy reported that the cfPWV and baPWV were positively associated with age and with $\mathrm{HbAlc}$, whereas femoral-ankle PWV was not. These studies support the observation that peripheral stiffness does not have the same associations with CAD, or its risk factors, that are observed for central AS $[26,30]$.

One hypothesis is that impaired cardiac autonomic function results in increased AS. An explanation could be that patients with CAN present more often with calcification of the tunica media of the arterial wall. It is noteworthy that the main determinant of the extent of arterial calcification is the severity of diabetic CAN. On the other hand, arterial calcification has been suggested as an important determinant of AS according to findings in humans and experimental models [14]. These data reveal that calcification of the arterial wall may be an additional common pathophysiological pathway that could explain the relationship between impaired cardiac autonomic function and AS. High sympathetic activity has been associated with AS in hypertensive patients with and without T2DM, as well as in healthy individuals. Increases in HR per se may lead to AS independently of changes in activity of the ANS [14].

The other hypothesis is that AS may lead to CAN via impairment of baroreceptor function induced by stiffening of the arterial wall. Several studies have found a significant association between low BRS and increased AS in patients with congestive HR, in older subjects. However, no difference in central BRS was observed among participants 
with abnormal and normal PWV. This finding may imply that DM per se is a strong factor affecting BRS and outweighs the potential effect of other factors on BRS [14].

Some data indicate that increased large AS appears already in prediabetic conditions. In a treatment-naïve and mostly "healthy" population from the ADDITION-Leicester cohort, cfPWV was increased in individuals with impaired fasting glucose or impaired glucose tolerance as compared to those with normal glucose metabolism, and the increase was identical to that of individuals with newly diagnosed T2DM. Indices of carotid stiffness increased with fasting plasma glucose, insulin and HbAlc; cfPWV increased with HbAlc; fasting glucose, HOMA-IR index and HbAlc, together with waist circumference, triglycerides and HDL-cholesterol were all predictors of cfPWV after a follow-up of 17 yrs. Finally, a recent study in middle-aged subjects free of CVD demonstrated the relationships of cfPWV with HOMA-IR index (direct) and telomere length (inverse) and suggested that IR linked with chronic inflammation can enhance telomere shortening (a marker of cellular senescence), and thus induce accelerated vascular aging [17].

\section{Conclusion}

It is reported that reduction of elasticity of large arteries and dysfunction of ANS appear already at the time of verification of DM. Questions arise: 1). What is the primary: vegetative dysfunction or increased rigidity of large arteries? 2). What cause-effect relationship exists between the dysfunction of the ANS and the rigidity of large arteries? Two hypotheses are proposed: AS of the arteries can lead to dysfunction of the myocardium, or, conversely, diabetic CAN promotes attachment and/or progression of the rigidity of the wall of the large arteries. In addition, the processes of reducing the elasticity of large arteries and CAN develop in parallel, taking into account the effects of aging and "toxic" effects of hyperglycemia [28].

One of the possible mechanisms that can explain the first hypothesis is the violation of the BRS, which are induced by increased AS. On the other hand, dysfunction of the cardiac ANS can change the elasticity of the artery wall, affecting the vascular tone of large arteries. In particular, animal experiments have shown that the integrity of the ANS plays an important role in preserving the elastic properties of the aorta [31]. However, it is known that experimental studies are accompanied by acute modulation of changes in the state of the ANS, while in patients with DM, a chronic, gradual, cumulative effect is observed. Another mechanism that may be involved in the processes of induction of AS and the development of autonomic dysfunction is the increase in HR. Indeed, an increase in HR per se, regardless of the changes in the activity of the ANS, contributes to the AS development. However, it was found that the normalization of HR in patients with T1DM does not affect the correlation between of left ventricular diastolic function and PWV. Consequently, the association between parasympathetic dysfunction and AS is not mediated by an increase in HR. Finally, changes in vegetative tone can increase the AS due to trophic effects, which contributes to changes in the structure of the vessels $[28,31]$.

A great amount of data demonstrates that hyperglycemia and IR activate number of mechanisms triggering the structural and functional changes in the arterial wall, which are likely to contribute to accelerated vascular aging and increased CV risk in T2DM [32-35]. Moreover, the stiffness gradient between the proximal elastic arteries and more distal muscular arteries decreases, and therefore, increases the transmission of pressure to the microcirculation that may be already damaged by diabetic microvascular disease. Moreover, T2DM is associated with other metabolic and systemic abnormalities, like atherogenic DLP, HT and obesity that may cause atherosclerosis, AS or both [17].

However, future work is needed to further clarify whether large AS and the pulsatile hemodynamic changes that accompany it are involved in the pathogenesis of DM, and whether interventions targeting AS are associated with improved clinical outcomes in diabetes mellitus.

\section{Conflicts of interest}

\section{None}

\section{References}

1. Ulleryd MA, Prahl U, BoErsbo J, Schmidt C, Nilsson S, et al. (2017) The association between autonomic dysfunction, inflammation and atherosclerosis in men under investigation for carotid plaques. PLoS One 12: e0174974. [Crossref]

2. Spallone V, Ziegler D, Freeman R, Bernardi L, Frontoni S, et al. (2011) Cardiovascular autonomic neuropathy in diabetes: clinical impact, assessment, diagnosis, and management. Diabetes Metab Res Rev 27: 639-653. [Crossref]

3. Ziegler D, Keller J, Maier C, Pannek J (2014) German diabetes association: Diabetic neuropathy. Exp Clin Endocrinol Diabetes 122: 406-415.

4. Serhiyenko VA, Serhiyenko AA (2015) Diabetic cardiac autonomic neuropathy: Do we have any treatment perspectives? World J Diabetes 6: 245-258. [Crossref]

5. Pop-Busui R, Boulton AJM, Feldman EL, Bril V, Freeman R, et al. (2017) Diabetic neuropathy: a position statement by the American Diabetes Association. Diabetes Care 40: $136-154$.

6. Mansour AS, Yannoutsos A, Majahalme N, Agnoletti D, Safar ME, et al. (2013) Aortic stiffness and cardiovascular risk in type 2 diabetes. J Hypertens 31: 1584-1592. [Crossref]

7. Bagherzadeh A, Nejati-Afkham A, Tajallizade-Khoob Y, Shafiee A, Sharifi F, et al (2013) Association of cardiac autonomic neuropathy with arterial stiffness in type 2 diabetes mellitus patients. J Diabetes Metab Disord 12: 55. [Crossref]

8. Agnoletti D, Mansour AS, Zhang Y, Protogerou AD, Ouerdane S, et al. (2017) Clinica interaction between diabetes duration and aortic stiffness in type 2 diabetes mellitus. $J$ Hum Hypertens 31: 189-194.

9. Xu M, Huang Y, Xie L, Peng K, Ding L, et al. (2016) Diabetes and risk of arterial stiffness: a Mendelian randomization analysis. Diabetes 65: 1731-1740. [Crossref]

10. De Oliveira Alvim R, Santos PCJL, Musso MM, de Cuhta R, Krieger JE, et al. (2013) Impact of diabetes mellitus on arterial stiffness in a representative sample of an urban Brazilian population. Diabetol Metab Syndr 5: 45. [Crossref]

11. Gaszner B, Lenkey Z, Illyés M, Sárszegi Z, Horváth IG, et al. (2012) Comparison of aortic and carotid arterial stiffness parameters in patients with verified coronary artery disease. Clin Cardiol 35: 26-31. [Crossref]

12. Okamoto M, Nakamura F, Musha T, Kobayashi Y (2016) Association between novel arterial stiffness indices and risk factors of cardiovascular disease. BMC Cardiovasc Disord 16: 211.

13. Chawla D, Bansal S, Banerjee BD, Madhu SV, Kalpa PO, et al. (2014) Role of advanced glycation end product (AGE)-induced receptor (RAGE) expression in diabetic vascular complications. Microvasc Res 95: 1-6.

14. Chorepsima S, Eleftheriadou I, Tentolouris A, Moyssakis I, Protogerou A, et al. (2017) Pulse wave velocity and cardiac autonomic function in type 2 diabetes mellitus. $B M C$ Endocr Disord 17: 27. [Crossref]

15. Ciftel M, Ertug H, Parlak M, Akcurin G, Kardelen F (2014) Investigation of endothelia dysfunction and arterial stiffness in children with type 1 diabetes mellitus and the association with diastolic dysfunction. Diab Vasc Dis Res 11: 19-25.

16. Zhao XY, Wang XF, Li L, Zhang L, Shen DL, et al. (2015) Effects of high glucose on human umbilical vein endothelial cell permeability and myosin light chain phosphorylation. Diabetol Metab Syndr 7: 98.

17. Kozakova M, Palombo C2 (2016) Diabetes mellitus, arterial wall, and cardiovascular risk assessment. Int J Environ Res Public Health 13: 201. [Crossref]

18. Barazzoni R, Zanetti M, Cappellari G, Semolic A, Boschelle M, et al. (2012) Fatty acids acutely enhance insulin-induced oxidative stress and cause insulin resistance by increasing mitochondrial reactive oxygen species (ROS) generation and nuclear factorkappa B inhibitor (Ikappa B)-nuclear factor-kappa B (NF-kappa B) activation in rat muscle, in the absence of mitochondrial dysfunction. Diabetologia 55: 773-782. 
19. van Dijk RAJM, Bakker SJL, Scheffer PG, Heine RJ, Stehouwer CDA (2003) Associations of metabolic variables with arterial stiffness in type 2 diabetes mellitus: focus on insulin sensitivity and postprandial triglyceridaemia. Eur J Clin Invest 33: 307-315. [Crossref]

20. Song MK, Davies NM, Roufogalis BD, Huang TH-W (2014) Management of cardiorenal metabolic syndrome in diabetes mellitus: a phytotherapeutic perspective. J Diabetes Res 2014: 313718

21. Cakar M, Balta S, Sarlak H, Akhan M, Demirkol S, et al. (2015) Arterial stiffness and endothelial inflammation in prediabetes and newly diagnosed diabetes patients. Arch Endocrinol Metab 59: 407-413. [Crossref]

22. Adamska A, Karczewska-Kupczewska M, Nikolajuk A, Otziomek E, Gorska M, et al. (2014) Relationships of serum soluble E-selectin concentration with insulin sensitivity and metabolic flexibility in lean and obese women. Endocrine 45: 422-429. [Crossref]

23. Mochizuki K, Inoue S, Miyauchi R, Misaki Y, Shimada M, et al. (2013) Plasma sEselectin level is positively correlated with neutrophil count and diastolic blood pressure in Japanese men. J Nutr Sci Vitaminol 59: 447-453.

24. Mancia G, Fagard R, Narkiewicz K, Redon J, Zanchetti A, et al. (2013) ESH/ESC guidelines for the management of arterial hypertension: the Task Force for the Management of Arterial Hypertension of the European Society of Hypertension (ESH) and of the European Society of Cardiology (ESC). Eur Heart J 34: 2159-219.

25. Bianchi L, Chiheb S, Banu I, Rezki A, Cosson E, et al. (2016) Influence of cardiac autonomic dysfunction and arterial stiffness on subendocardial myocardial viability in patients with type 2 diabetes. Diabetes Metab 42: 297-298

26. Loehr LR, Meyer ML, Poon AK, Selvin E, Palta P, et al. (2016) Prediabetes and Diabetes Are Associated With Arterial Stiffness in Older Adults: The ARIC Study. $A m$ J Hypertens 29: 1038-1045. [Crossref]
27. Yeboah K, Antwi DA, Gyan B (2016) Arterial stiffness in nonhypertensive Type 2 Diabetes patients in ghana. Int J Endocrinol 2016: 6107572. [Crossref]

28. Christen AI, Armentano RL, Miranda A, Graf S, Santana DB, et al. (2010) Arterial wall structure and dynamics in type 2 diabetes mellitus methodological aspects and pathophysiological findings. Curr Diabetes Rev 6: 367-377. [Crossref]

29. Tsuchikura S, Shoji T, Kimoto E, Shinohara K, Hatsuda S, et al. (2010) Central versus peripheral arterial stiffness in association with coronary, cerebral and peripheral arterial disease. Atherosclerosis 211: 480-485.

30. Meyer ML, Tanaka H, Palta P, Cheng S, Gouskova N, et al. (2016) Correlates of segmental pulse wave velocity in older adults: the Atherosclerosis Risk in Communities (ARIC) Study. Am J Hypertens 2016; 29:114-122.

31. Stoner L, Young JM, Fryer S (2012) Assessments of arterial stiffness and endothelial function using pulse wave analysis. Int J Vasc Med 2012: 903107. [Crossref]

32. Plenner SB, Chirinos JA (2015) Arterial stiffness in diabetes mellitus. Atherosclerosis 238: $370-379$.

33. Smulyan H, Lieber A, Safar ME (2016) Hypertension, Diabetes Type II, and Their Association: Role of Arterial Stiffness. Am J Hypertens 29: 5-13. [Crossref]

34. Ecobici M, Stoicescu C (2017) Arterial Stiffness and Hypertension - Which Comes First? Maedica (Buchar) 12: 184-190. [Crossref]

35. Elias MF, Crichton GE, Dearborn PJ, Robbins MA, Abhayaratna WP (2017) Associations between type 2 diabetes mellitus and arterial stiffness: a prospective analysis based on the Maine-Syracuse Study. Pulse 5: 88-98. [Crossref]

Copyright: $(02018$ Serhiyenko VA. This is an open-access article distributed under the terms of the Creative Commons Attribution License, which permits unrestricted use, distribution, and reproduction in any medium, provided the original author and source are credited. 